\title{
Research on the Architecture of a Basic Reconfigurable Information Communication Network
}

\author{
Ruimin Wang, ${ }^{1}$ Lei Zhuang, ${ }^{1}$ Yuqiang Luo, ${ }^{2}$ and Hongchang Chen ${ }^{3}$ \\ ${ }^{1}$ School of Information Engineering, Zhengzhou University, Zhengzhou 450001, China \\ ${ }^{2}$ Informatization Office, University of Shanghai for Science and Technology, Shanghai 200093, China \\ ${ }^{3}$ National Digital Switching System Engineering \& Technological R\&D Center, Zhengzhou 450002, China \\ Correspondence should be addressed to Ruimin Wang; iermwang@zzu.edu.cn and Yuqiang Luo; abel.luo.me@gmail.com
}

Received 20 September 2013; Accepted 6 November 2013

Academic Editor: Guoliang Wei

Copyright (c) 2013 Ruimin Wang et al. This is an open access article distributed under the Creative Commons Attribution License, which permits unrestricted use, distribution, and reproduction in any medium, provided the original work is properly cited.

The current information network cannot fundamentally meet some urgent requirements, such as providing ubiquitous information services and various types of heterogeneous network, supporting diverse and comprehensive network services, possessing high quality communication effects, ensuring the security and credibility of information interaction, and implementing effective supervisory control. This paper provides the theory system for the basic reconfigurable information communication network based on the analysis of present problems on the Internet and summarizes the root of these problems. It also provides an in-depth discussion about the related technologies and the prime components of the architecture.

\section{Introduction}

The Internet has become one of the most important infrastructures supporting economic society development, social progress, and technological innovation, which is the important symbol that indicates the national basic power and economic competitiveness of a country. With the popularization of the Internet and the continued emergence of new application, such as data-hungry devices, mobile access, ubiquitous network, pervasive computing, and heterogeneous environment, the Internet is confronted with unprecedented technical challenges in manageability, extendibility, security, high performance, mobility, instantaneity, and so forth.

To overcome these technical challenges, the developed countries, such as the USA, have begun to study the nextgeneration network from the middle 1990s [1]. With the progress of research on this issue, a great deal of literature has appeared see, for example, [2-5]. Chinese scientists and engineers have also turned to study next-generation network from the late 1990s. For now, researches on the key technologies and standards, the large-scale experimental network and the basic theory for the next-generation network have made considerable progress. At the same time, people have a deep understanding of the long-term, arduous, and complex nature of the study about the next-generation network. One after another, the developed countries have included the nextgeneration network in the key development orientation of information technology.

In the first section, the present situation of today's Internet was analyzed, and some problems are being pointted out; the third section explored the basic theory and key technology of a basic reconfigurable information communication network. In the end, the properties of the reconfigurable network are analyzed.

\section{Current Actualities and Problems of Internet}

From the perspective of the architecture and mechanism of basic network, current information communication network cannot fundamentally satisfy some urgent requirements such as providing ubiquitous information services and various types of heterogeneous network, supporting diverse and comprehensive network services, possessing high quality communication effects, ensuring the security and credibility of information interaction, and implementing effective supervisory control [6]. On the one hand, a common basic carry 
mechanism of current information network, the function of $\mathrm{TCP} / \mathrm{IP}$, is oversimple, and it leads to a huge gap between the basic ability of network and the requirement of upper-layer application. On the other hand, over past decades, people's efforts of improving network to fill this gap are based on the limited TCP/IP capacity. Therefore, it is bound to lead to this kind of enhancement which is patchwork, which cannot blend with the core function of network effectively. This kind of weak core architecture naturally leads to low basic capacity of information network, and it is unable to guarantee some high-level requirements [7]. Specifically solving the following two problems becomes imperative.

(1) Necessary of research on principal theory of basic information communication network: summing up the developmental trajectory of study on the information network in the world, it is easy to find that there are some engineering technical undertones, tinkering with the existing information network architecture or a simple extension which can't fundamentally meet some requirements, for example, ubiquity, interconnection, integration, heterogeneity, reliability, manageability, and extendibility. However, taking the enhancement of the basic ability of an network as the starting point is a effective breakthrough. A fundamental innovation of network architecture is the key to solve this problem.

The basic IP network occupies a leading position in current information network, whereas the structure of which is simple with weak function. As the IP network has become the most important part of the national information infrastructure, the simplicity which has not changed for several decades is seriously insufficient to support ubiquitous information services, various and all-around network service, quality assurance communication effect, safe and reliable information interaction, and so forth. The gap between the unchanged basic ability of network and people's increasing requirement for network has seriously impacted the national information security and industry's sustainable development for the national economy and people's living.

(2) It is necessary to embed some new types of function elements into the core of architecture, which will naturally cooperate and work with each other, and the bottleneck restriction to the IP network can be avoid. The current IP network still mainly relies on connectionless packet, and the interconnected transmission capacity of which is a significant bottleneck constraining its overall capability. The structure of basic network is ossified and the core part is short of intellectualization, which leads to low capacity of security, controllability, adaptability, extendibility, and evolving ability. That is because the IP network is limited by the initial design philosophy and affected by its development history, which leads to a huge contrast between the rich and multiplex services in the upper and lower layer and the simple and the rigescent structure of basic network. The IP network does not possess basic intelligence of enhancing its ability, and cannot cope with urgent challenging problems. However, there exist some drawbacks in the evolution scheme, for example, the single function of network layer, the excessive separation between service and network, poor ability of network collaboration, and so forth. These defects make the current basic network hard to achieve reliability, manageability, extendibility, and so forth.

\section{Theoretical System of the Basic Reconfigurable Information Communication Network}

From the above analysis of the current information network, the disadvantages of the basic IP network are obvious. Therefore, for the purpose of solving the challenging problem encountered in the IP network (e.g., performance, security, multicast, mobility, extendibility, etc.), it is necessary to construct a new architecture of network. In the past decades, the study of reconfigurable networks has been paid much attention from many researchers, and a great number of results have been reported in the literature, see, for example, [8-14] and the reference therein. In this paper, we propose a theory of the basic reconfigurable information communication network from a new perspective, which can be divided into three parts: network metaability theory, polymorphic addressing and routing mechanism, and reconfiguration mechanism. With the architecture proposed in the paper, the requirements of ubiquity, interconnection, quality, integration, heterogeneity, reliability, controllability, manageability, extendibility, and so forth can be addressed. The main contribution of this paper is mainly duplex: (1) according to the fundamental nature of the future basic information communication network, the ideas of constructing a reconfigurable and extendable physical network are given; (2) by adapting the metaservice and metaability, the network can pervade various businesses based on the network addressed in this paper. Consequently, the customizable and required network service will be provided.

3.1. Network MetaAbility Theory. The purpose of network metaability theory is to introduce a new kind of transmission ability into the information communication network and establish a theoretical foundation for the architecture of the basic reconfigurable information communication network. The implementation model of the network metaability abstracts and divides the network resource on three levels of business, metaservice, and metaability from the reconfiguring perspective. At the same time, as shown in Figure 1, it describes the network resource on three levels of point metaability, face metaability, and metaservice in a multiscale way.

Point metaability is a logical entity obtained by reconfiguring and abstracting the heterogeneous and distinctive resources in the network nodes, which provides the basic carry for reconfiguring the whole network by optimally scheduling and programming the node resources. From the whole network perspective, the face metaability perceives service cluster, assembles the behavior characteristics of the network resources in data layer, and adapts metaservice to pervade various services.

The structure form of the reconfigurable network based on the metaability manifests as the flexible adaptive capacity to the various modal characteristics of the macrocircuit 


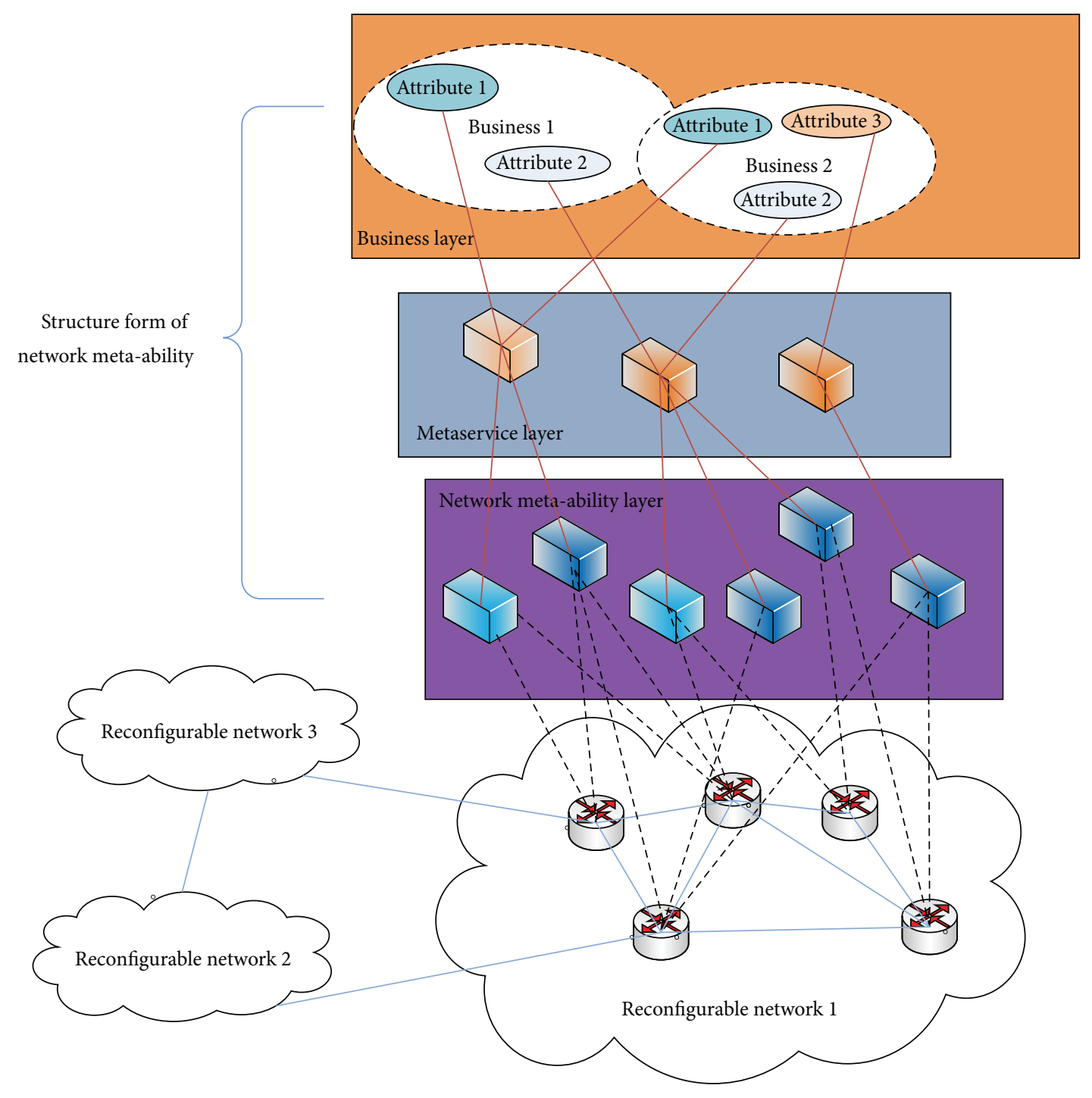

FIGURE 1: Structure form of reconfigurable network based on the metaability.

which is a functional element of the metaability. For a set of same performance, the metaability responses positively, selectively construct and reuse macrocircuit with qualified performance requirements by perceiving the characteristic and dynamic behavior of the node resource and network resource and finally satisfy the carrying and service requirements for various services.

By modeling metaservice and metaability regularly, the logical mapping relationship between business, metaservice, and metaability can be established; and with further canonical modeling of point metaability, a unified control management in the management layer can be realized by defining standard interface. For a heterogeneous and interconnected network in physics, management layer can implement reconfiguration operation to a whole network and configure a bearer network to support different service.

Two elements of the metaability layer in the network metaability can form an internal logical relationship of trusting support and united effect. Packet transport capacity directly supports survivability and robustness of a network, and hence the interconnected transmission ability inherited from the basic network constitutes the material foundation of the macrocircuit and the reconfigurable network. Based on the packet capacity, macrocircuit has a new connotation of the interconnected transmission ability in the innovative information communication network and constitutes a kind of enhanced basic capability; at the same time, macrocircuit and packet capacity become a supporting element of the reconfigurable ability.

Structure form of the metaability based on business, metaservice, point metaability, and face metaability makes some demands on reconfigurable network at various levels. In terms of metaservice, it can reduce the coupling between network resource and business by assembling and abstracting common characteristic of various services. Resorting to basic carrying capacity provided by the face metaability over the whole network, metaservice can adapt various services by reconfiguring. As shown in Figure 2, the process of 


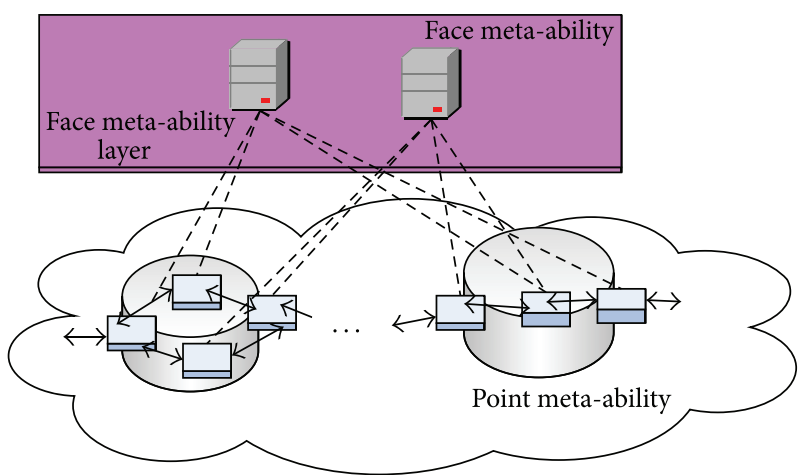

FIgURE 2: The reconfiguration mechanism of face metaability.

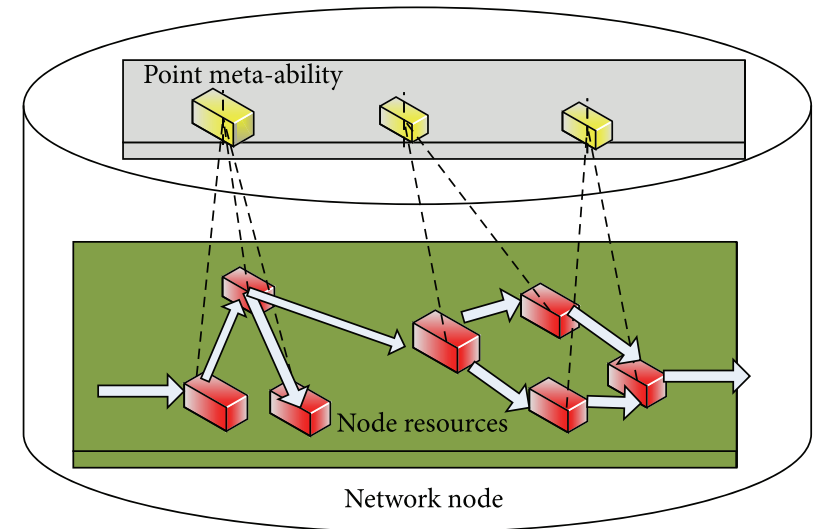

FIGURE 3: The reconfiguration mechanism of point metaability.

reconfiguring manifest as the realization of the pervasive services by the mutual assistance between the nodes in the whole network and the flexible combination of the internal resources.

Support of face metaability for metaservice is all-around as shown in Figure 2, some possible situations include; (1) a metaservice needs to assemble several face metaabilities to support business; (2) one face metaability may provide more than one metaservice at some points. Therefore, every node used to support face metaability fully considers their mutual coordination and the resource sharing. From the whole network perspective, face metaability perceives service characteristic, assembles, and matches network resource behavior of data layer so as to reconfigure the metaservice. The reconfiguration of the face metaability manifests as the process of integrating point metaability to adapt metaservice.

As shown in Figure 3, it will provide the best configuration scheme of the node resources for deploying point metaability by optimal scheduling and programming of the node resources.

Point metaability is the smallest granularity of resource and ability in the basic reconfigurable network architecture, which provides the basic carry for reconfiguring the whole network. Reconfiguration of point metaability refers to the reprogramming control of the software or hardware resources in nodes (e.g., node protocol entity, form management, control entity, network processor microkernel, etc.) or a full reload of software to reconfigure these resource modules flexibility.

Performance of control channel of point metaability has great significance for reconfigurability of the whole network. It can analyse traffic in control channel of the point metaability by the model of traffic matrix estimation or other method, and the distribution of the traffic in the control channel can be estimated completely, which provides evidence for the design of control channel to optimize traffic in the control channel. Given the traffic in the control channel as $X(t)$, then the multiscale analysis on $N$ order is

$$
\begin{aligned}
\gamma(t) & =\left[\begin{array}{c}
\left(X(t)_{L}^{M}\right) \\
\left(X(t)_{H}^{M}\right) \\
\cdots \\
\left(X(t)_{H}^{N-2}\right) \\
\left(X(t)_{H}^{N-2}\right)
\end{array}\right]=\left[\begin{array}{c}
H^{M} H^{M+1} H^{M+2} \cdots H^{N-1} \\
G^{M} H^{M+1} H^{M+2} \cdots H^{N-1} \\
\cdots \\
G^{N-2} H^{N-1} \\
G^{N-1}
\end{array}\right] \\
& =X(t)^{N},
\end{aligned}
$$

where $H^{i}$ and $G^{i}$ are the scale coefficient of multiscale analysis and wavelet coefficients, respectively. The estimate of $\widehat{\gamma}(t)$, namely, the value of traffic matrix, can be obtained by Kalman filter algorithm after wavelet transformation.

3.2. Polymorphic Addressing and Routing Scheme. Polymorphic addressing and routing mainly solve the problem of network addressing, routing, and switching which is driven by metaservice and supports various network architectures.

Basic protocol is core network layer protocol in the basic reconfigurable network. In the TCP/IP network, IP address is designed for location marker and identity of the endpoint, and this kind of dual role does not only limit mobility of the network but also brings the security issue. Design of the basic protocol provides a solution to separate the embedded identity and the address. With the change of service requirement from communication to data content, the new basic protocol must effectively support data-oriented addressing and routing.

Polymorphic protocol which is generated by the basic protocol has various working patterns, and it can be expressed as a different protocol architecture by specializing the basic protocol or different working patterns of one protocol architecture. The relationship between the basic protocol and the polymorphic protocol is shown in Figure 4 .

As a crucial support for the network addressing and routing, reconfiguration makes network function and behavior change dynamically according to users' different needs or switch between different protocol architectures and running states of a same protocol architecture. By reconfiguring, it can form a number of logical bearer network and provide basics for isolating metaservice and resource allocation. In the polymorphic addressing and routing, the IP packet forwarding is realized in one or more logical bearer networks. Several different protocols can be executed simultaneously in a same physical network, which can provide different 

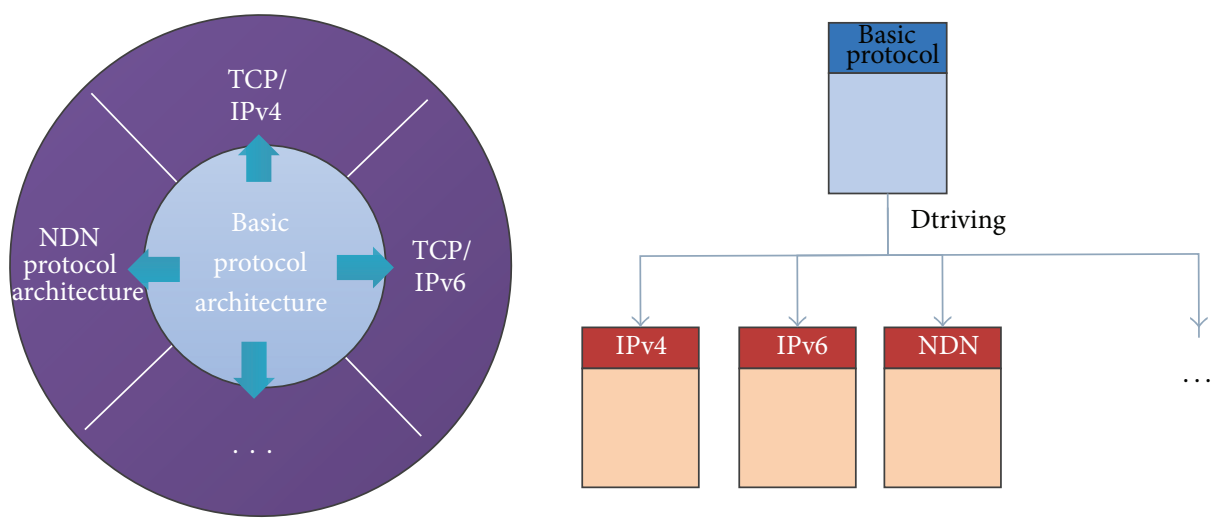

FIGURE 4: The basic protocol architecture and the polymorphic protocol architecture.
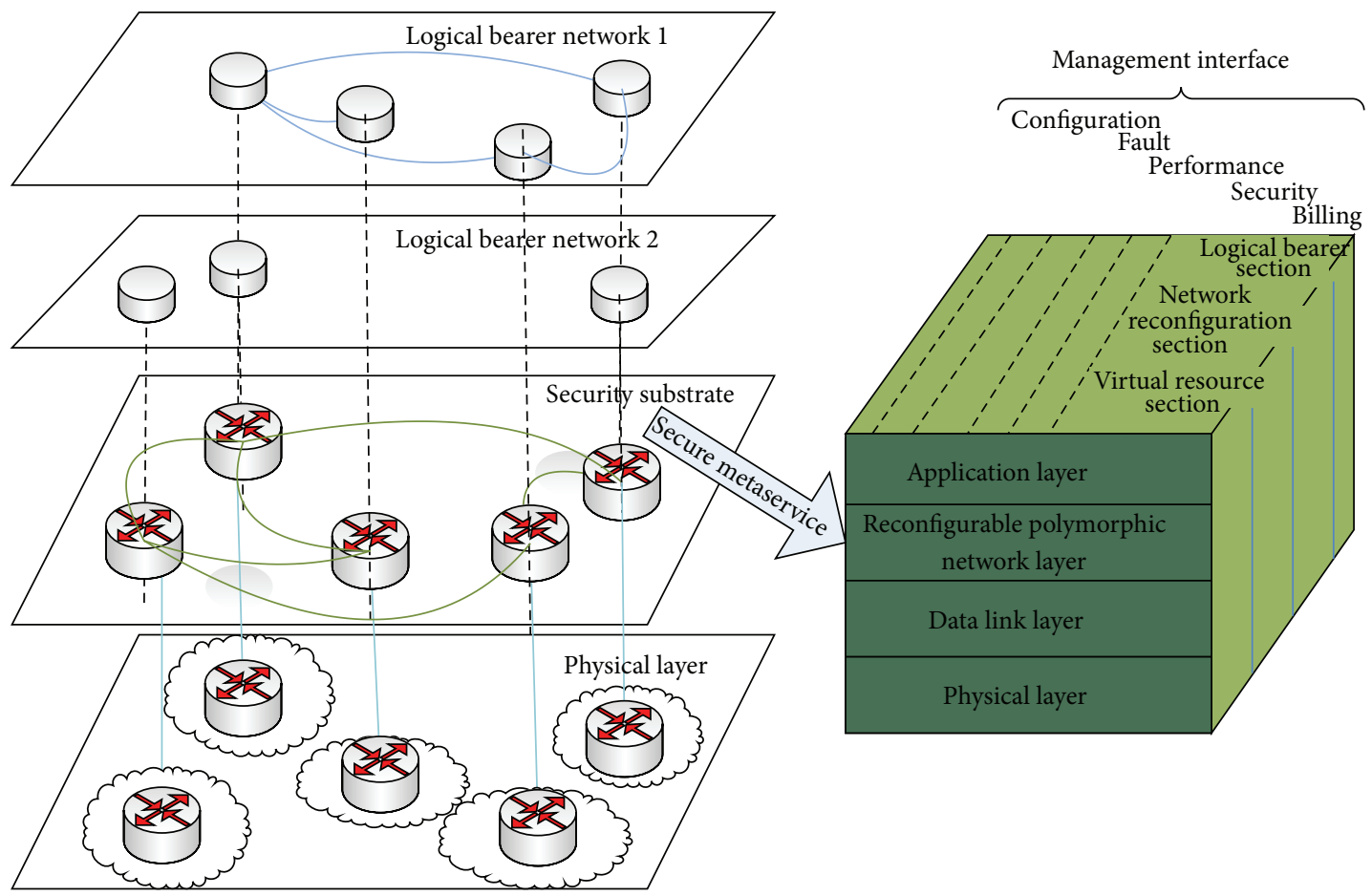

FIGURE 5: The logical bearer network's structure form based on security substrate.

metaservice ability for services with different characteristics in the form of bearer network.

3.3. Mechanism of Reconfiguring Network. The basic reconfigurable information communication network, which is manageable and controllable architecture, has the mechanism of security substrate and structure of multilevel intensity based on the safe, manageability, and controllability mechanism of reconfiguring. By the behavior oriented situation analysis, it can solve the tracing problem of security behaviour and fully guarantee user security, network security, and service security in the basic reconfigurable information communication network, which can realize the real-time sensing, warning, and handing of situation.

The structure form of the logical bearer networks based on security substrate is shown in Figure 5. Security substrate is the sum of basic security element and function constructed for common security and control characteristic requirement. Different security substrates have different requirements of security strength level. Logical bearer network, which aims at satisfying bearer requirement of service completely and adapting network security requirement, provides security assurance for networks, services and users by utilizing the 
network metaability with the packet transport, reconfigurability, and network metaability on the basis of the core element of macrocircuit, while providing the best service by making the most of the network resources.

\section{Analysis of Theoretical System of the Basic Reconfigurable Information Communication Network}

(1) In the third section, the main research target, research approach, and specific framework module of the theoretical system of the basic reconfigurable information communication network are described, which include metaability theory, polymorphic addressing and routing mechanism, and network reconfiguration mechanism. It fundamentally breaks through the capacity bottleneck of the traditional IP network and solves many problems, such as poor transmission capacity of the basic traditional information network, low expansibility of service adaptation, and low controllability and manageability.

(2) Based on the theory of the basic reconfigurable information communication network, it can construct a functional reference model of improving the network layer and transport layer to solve the functional bottleneck problem of the network layer in the current IP network, so as to match the increasing application requirements and abundant transmission resources.

(3) Based on the reconfiguration mechanism of pervasive service and the built approach of logical bearer network, the flexible adjustment of the network structure and function can be realized, and the polymorphism can be presented by assembling node ability and network resource, which can satisfy different functional requirements of various services.

(4) The mechanism of addressing, routing, and switching of basic reconfigurable network provides the basal guarantee for the same operation in new type of network, which makes the network be expected to support security, expansibility, and mobility.

(5) By establishing the structure and mechanism of security and control, it can ensure the basic attributes of the new type of network, such as network security, information security, users security, controllability, and manageability.

\section{Conflict of Interests}

The authors declare that there is no conflict of interests regarding the publication of this paper.

\section{Acknowledgments}

This work was supported in part by the National Grand Fundamental Research 973 Program of China under Grant no. 2012CB315901, the Key Project of Technology Department of Henan Province of China under Grant 122102210042, and the Scientific and Technological Brainstorm Project of Henan Province of China under Grant 12B520054.

\section{References}

[1] J.-P. Wu and K. Xu, "Research on next-generation internet architecture," Journal of Computer Science and Technology, vol. 21, no. 5, pp. 723-731, 2006.

[2] C.-S. Lee and D. Knight, "Realization of the next-generation network," IEEE Communications Magazine, vol. 43, no. 10, pp. 34-41, 2005.

[3] M. N. O. Sadiku and T. H. Nguyen, "Next generation networks," IEEE Potentials, vol. 21, no. 2, pp. 6-8, 2002.

[4] H. M. Sigurdsson, S. E. Thorsteinsson, and T. K. Stidsen, "Cost optimization methods in the design of next generation networks," IEEE Communications Magazine, vol. 42, no. 9, pp. 118-122, 2004.

[5] J. Simoes and S. Wahle, "The future of services in next generation networks," IEEE Potentials, vol. 30, no. 1, pp. 24-29, 2011.

[6] Z. Zhang, B. Q. Wang, and J. Liu, "Balanced counting Bloom filters: a space-efficient synoptic data structure for a highperformance network," IET Communications, vol. 6, no. 15, pp. 2259-2266, 2012.

[7] H. Ma, Y. Guo, D. Cheng, and J. Zhang, "Reducing burst packet loss through route-free forwarding," Journal of Electronics, vol. 27, no. 3, pp. 363-370, 2010.

[8] I. Artundo, W. Heirman, C. Debaes, M. Loperena, J. Van Campenhout, and H. Thienpont, "Low-power reconfigurable network architecture for on-chip photonic interconnects," in Proceedings of the 17th IEEE Symposium on High Performance Interconnects (HOTI '09), pp. 163-169, New York, NY, USA, August 2009.

[9] M. Attig and J. Lockwood, "A framework for rule processing in reconfigurable network systems," in Proceedings of the 13th Annual IEEE Symposium on Field-Programmable Custom Computing Machines (FCCM '05), pp. 225-234, April 2005.

[10] C. A. Balanis, "Smart antennas for future reconfigurable wireless communication networks," in Proceedings of the IEEE Topical Conference on Wireless Communication Technology, pp. 181-182, October 2003.

[11] C. Y. Chao and M. Ilyas, "Fast reconfigurable communication networks," in Proceedings of the 8th Annual International Phoenix Conference on Computers and Communications, pp. 248-252, Scottsdale, Ariz, USA, March 1989.

[12] C. Y. Chao and M. Ilyas, "Distributed channel allocation in reconfigurable communication networks," in Proceedings of the 13th Annual IEEE Symposium on Field-Programmable Custom Computing Machines, pp. 896-900, Columbia, SC, USA, April 1989.

[13] J.-C. Chen, J.-H. Yeh, S.-H. Hung, F.-C. Chen, L.-W. Lin, and Y.W. Lan, "Reconfigurable architecture and mobility management for next-generation wireless IP networks," IEEE Transactions on Wireless Communications, vol. 6, no. 8, pp. 3102-3113, 2007.

[14] S. Yusuf, W. Luk, M. Sloman, N. Dulay, E. C. Lupu, and G. Brown, "Reconfigurable architecture for network flow analysis," IEEE Transactions on Very Large Scale Integration (VLSI) Systems, vol. 16, no. 1, pp. 57-65, 2008. 


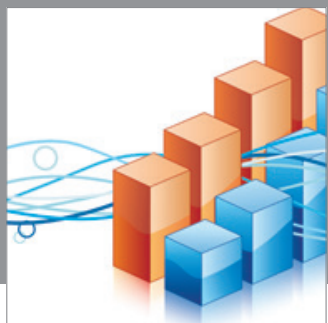

Advances in

Operations Research

mansans

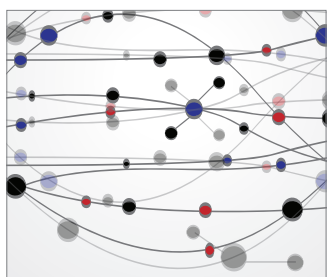

The Scientific World Journal
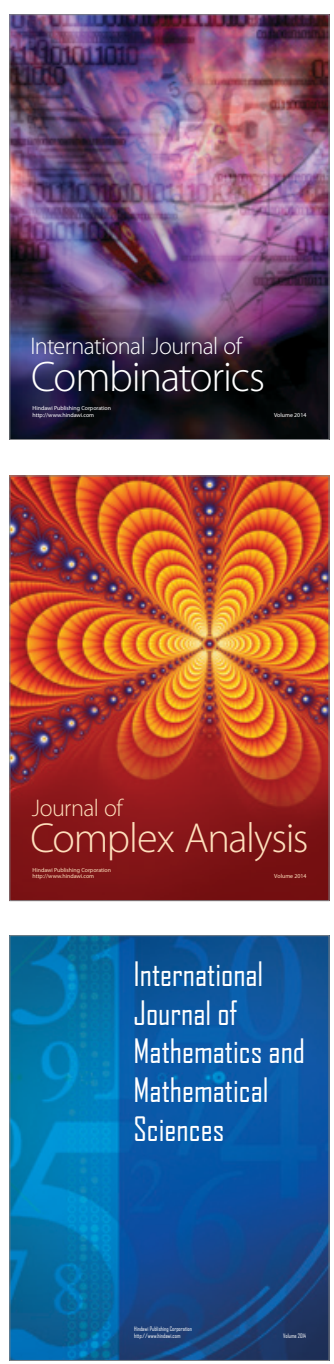


Submit your manuscripts at http://www.hindawi.com
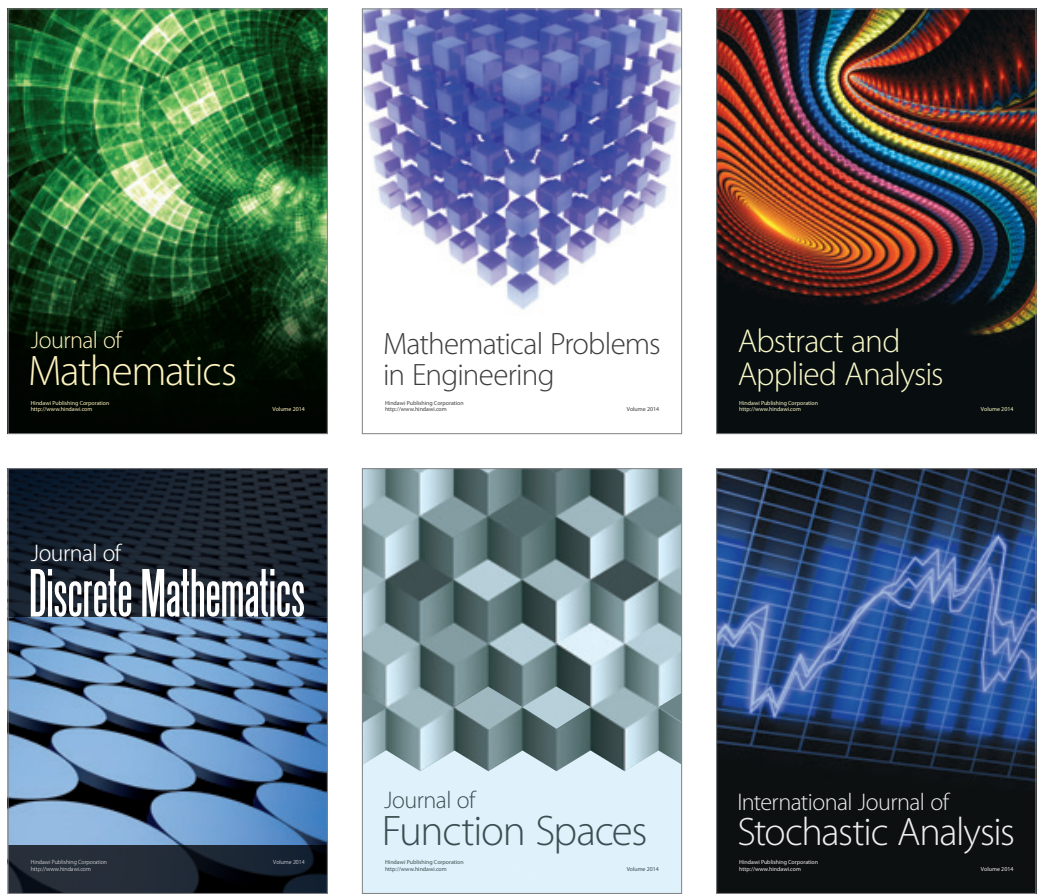

Journal of

Function Spaces

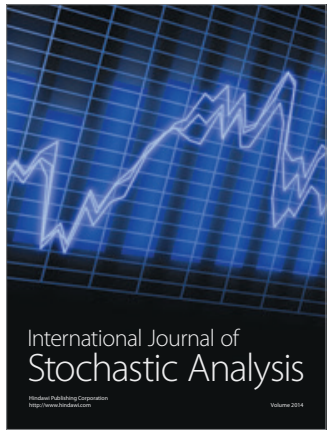

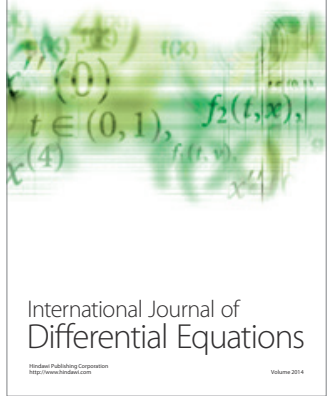
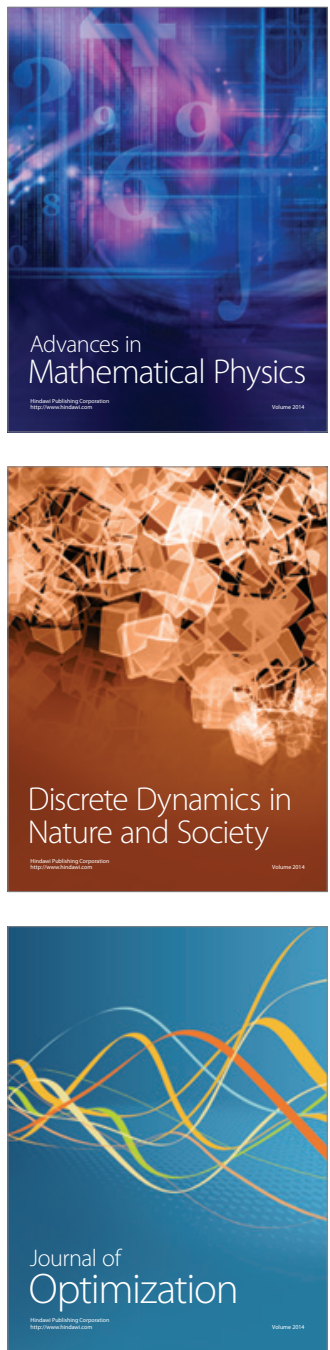